\title{
Una mirada alternativa para el tratamiento de camuflaje de la clase III esquelética. Reporte de un caso clínico
}

\section{An alternative point of view for the camouflage treatment of a skeletal class III. A clinical case report}

\author{
Orlando Enrique Martínez-Bermudez ${ }^{1}$ (D) , Jaime Enrique Plazas-Román² (iD), Cesar Agustín Vergara-Guerra ${ }^{3}$ (iD) , Antonio \\ Díaz-Caballero ${ }^{4}$ iD
}

1. Corporación Universitaria Rafael Núñez. Cartagena, Colombia. Correo: orlandoemartinez@gmail.com - https://orcid.org/0000-0002-5347-1039 2. Corporación Universitaria Rafael Núñez. Cartagena, Colombia. Correo: jaime.plazas@curnvirtual.edu.co - https://orcid.org/0000-0002-5040-6899 3. Corporación Universitaria Rafael Núñez. Cartagena, Colombia. Correo: cesarvergarag@hotmail.com - https://orcid.org/0000-0002-4580-3785 4. Universidad de Cartagena. Cartagena, Colombia. Correo: adiazc1@unicartagena.edu.co - https://orcid.org/0000-0001-9693-2969

Tipología: Reporte de caso clínico

Para citar este artículo: Martínez-Bermudez O, Plazas-Román JE, Vergara-Guerra CA, Díaz-Caballero A. Una mirada alternativa para el tratamiento de camuflaje de la clase III esquelética. Reporte de un caso clínico. Duazary. 2020 abril; 17(2): 58 - 65. Doi: http://dx.doi.org/10.21676/2389783X.3236

Recibido en diciembre 29 de 2017

Aceptado en agosto 13 de 2019

Publicado en línea en diciembre 02 de 2019

\section{RESUMEN}

\section{Palabras clave:} ortodoncia; maloclusión Angle clase III; diente molar; cirugía bucal; dentición mixta.

\section{Keywords:} Orthodontic Brackets; malocclusion, Angle class iii; Molar; Tooth Extraction; Mixed
El tratamiento de las maloclusiones clase III esqueléticas es un gran reto para el equipo odontológico, especialmente en niños o adolescentes, de tal manera que es importante contar con alternativas que no sean las utilizadas de forma tradicional. El propósito de este reporte es revisar el tratamiento ortodóntico interceptivo de una paciente femenina con maloclusión clase III en dentición mixta y apiñamiento severo en ambas arcadas con disminución en el espacio para la erupción de caninos, quien fue tratada desde los nueve años con extracción de primeros molares mandibulares. Se presentan las bases del tratamiento y su resultado a corto plazo. Se discuten algunos factores importantes para establecer un camuflaje de una relación molar de clase III y además revisar las limitaciones del tratamiento con la finalidad de prevenir la necesidad de una cirugía ortognática.

\section{ABSTRACT}

Skeletal class III treatment is a challenge for the dental team, especially in kids and teenagers; therefore, it is important to have multiple alternative and treatment modalities that are not traditionally used. The purpose of this report is to review the interceptive orthodontic treatment of a female patient with a Class III malocclusion in the mixed dentition, severe crowding in both arches with no space for upper and lower canines' eruption. The patient was treated at nine years old, nonsurgical with extraction of mandibular first molars. The basis for this treatment approach is presented, and the short term treatment results reviewed. Important factors to consider when establishing a camouflage of a Class III molar relationship and its limitations are discussed in order to avoid an orthognatic surgery. 


\section{INTRODUCCIÓN}

La clase III esquelética es una maloclusión que se presenta de manera frecuente en diversos centros de atención en diferentes ciudades del orbe, incluyendo Colombia con su diversidad poblacional ${ }^{1,2}$. Este tipo de maloclusión se convierte en un reto clínico para el ortodoncista que busca lograr resultados satisfactorios sin tener que acudir a procedimientos invasivos como una cirugía ortognática, teniendo en cuenta que las maloclusiones de clase III empeoran a medida que el individuo aumenta en edad ${ }^{3}$. La intervención temprana de esta anomalía se encuentra asociada con una mejor conformidad para el paciente y una mejor respuesta ortopédica; sin embargo, el tratamiento en la dentición mixta o permanente temprana puede producir resultados más favorables ${ }^{4}$.

El ortodoncista prefiere la intervención en la dentición mixta y aun en dentición temporal para la corrección de la clase III, donde combina la utilización de aparatos funcionales, máscaras faciales y hasta craneomaxilares invertidas ${ }^{4}$; $\sin$ embargo, no todos los casos logran resultados satisfactorios con este tipo de terapias. Durante la dentición mixta tardía o permanente temprana existen casos de maloclusiones clase III donde existe la posibilidad de tratar a estos pacientes mediante camuflaje ortodóntico preservando el perfil facial ${ }^{5}$, también llamados casos límites (border line). Estos casos tienen sus limitaciones y dependen del grado de severidad y edad del paciente para su corrección $n^{6,7}$.

A la clínica de una facultad de Odontología de la ciudad de Cartagena se presentó una niña adolescente con clasificación esquelética de clase III, cuya familia no contaba con las posibilidades económicas para practicarle una cirugía ortognática a futuro como tratamiento de su maloclusión. Aunque no existe una total aprobación como tratamiento ortodóntico, la extracción de primeros o segundos molares inferiores para la corrección de las relaciones de maloclusión de clase III son documentadas en la literatura ${ }^{8,9}$, y por esto fue propuesta a la paciente como una alternativa para su corrección.

Dentro de las consideraciones negativas por tener en cuenta antes de establecer esta terapia como plan de tratamiento se encuentran la ausencia de terceros molares, terceros molares pequeños o malformados, terceros molares en posición horizontal, agenesias congénitas de premolares o incisivos inferiores. Aunque es prematuro conocer los resultados a largo plazo del presente caso, un correcto diagnóstico que conlleve objetivos específicos de tratamiento puede ayudar a corregir este tipo de anomalía en pacientes en crecimiento activo ${ }^{10}$.

\section{REPORTE DE CASO}

Paciente femenina de nueve años con $32 \mathrm{~kg}$ de peso, en dentición mixta, con crecimiento activo sin historia previa de tratamiento ortopédico u ortodóntico y cuyo motivo de consulta es una revisión odontológica de rutina. La madre refiere que el padre de la paciente presenta características faciales similares.

\section{Hallazgos}

Tipo facial leptoprosopo, simétrica, perfil recto, clase III esquelética, prognatismo del maxilar inferior, vestibuloversión de incisivos superiores, linguoversión de incisivos inferiores, clase III molar de Angle, erupción prematura de premolares superiores asociada a pérdida temprana de molares temporales por caries, apiñamiento severo en ambas arcadas con disminución en el espacio para la erupción de caninos, sobremordida vertical y horizontal al límite, línea media dental inferior desviada hacia la izquierda, mordida cruzada posterior bilateral. Radiográficamente se observa formación completa de la corona de los terceros molares tanto superiores como inferiores (figura1). 

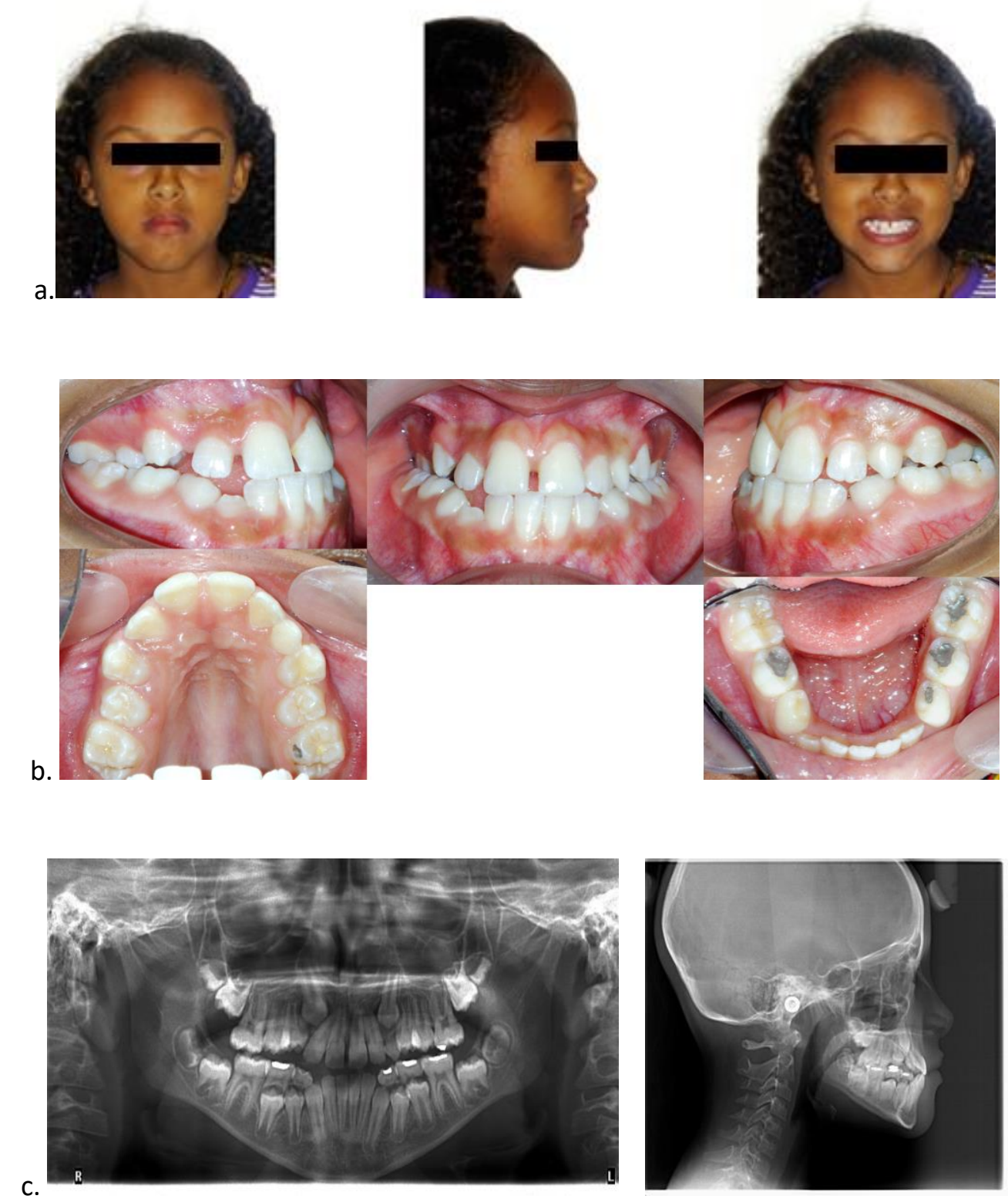

Figura 1. Estudios iniciales.

a. Fotografías faciales de frente, perfil y sonrisa pretratamiento a la edad de nueve años; b. Fotografías intraorales donde se aprecian la maloclusión clase III de Angle y el apiñamiento severo en ambas arcadas para la erupción de los caninos superiores e inferiores; c. Radiografías panorámica y de perfil al inicio del tratamiento.

\section{Análisis cefalométrico}

Se obtuvieron radiografías pretratamiento que luego se trazaron con el programa Cefax. Se tomaron las medidas tradicionales de Steiner y McNamara ${ }^{11}$, y de este modo se encontraron las siguientes medidas de importancia: SNA $\left(83^{\circ}\right)$, SNB $\left(84^{\circ}\right)$, ANB $(-1)$, Wits $(-5)$, 1Sup-SN $\left(114^{\circ}\right)$, 1Sup-PP $\left(120^{\circ}\right)$, 1Inf-PM $\left(84^{\circ}\right)$, eje de crecimiento $\left(90^{\circ}\right)$.

\section{Plan de tratamiento}

Los objetivos del tratamiento fueron:

1. Aumentar la sobremordida vertical, disminuyendo la posibilidad de una mordida cruzada anterior durante el crecimiento de la paciente.

2. Aumentar el perímetro de arco, con la finalidad de lograr espacio para la erupción de caninos superiores mediante expansión del arco superior. 
3. Lograr espacio para erupción de caninos inferiores dentro del arco sin producir cambios en la angulación de los incisivos inferiores.

\section{Tratamiento efectuado}

El caso inicia colocando una placa ortopédica maxilar tipo Hawley con tornillo de expansión en el arco superior, realizando una expansión lenta según el protocolo de Baccetti et al. ${ }^{12}$. Luego de firmar el consentimiento informado por parte de la madre de la paciente, donde se le comunican las expectativas y los objetivos del tratamiento, se hacen extracciones de primeros molares en el arco inferior, esperando la erupción de segundo y tercer molares. (figura 2). a.

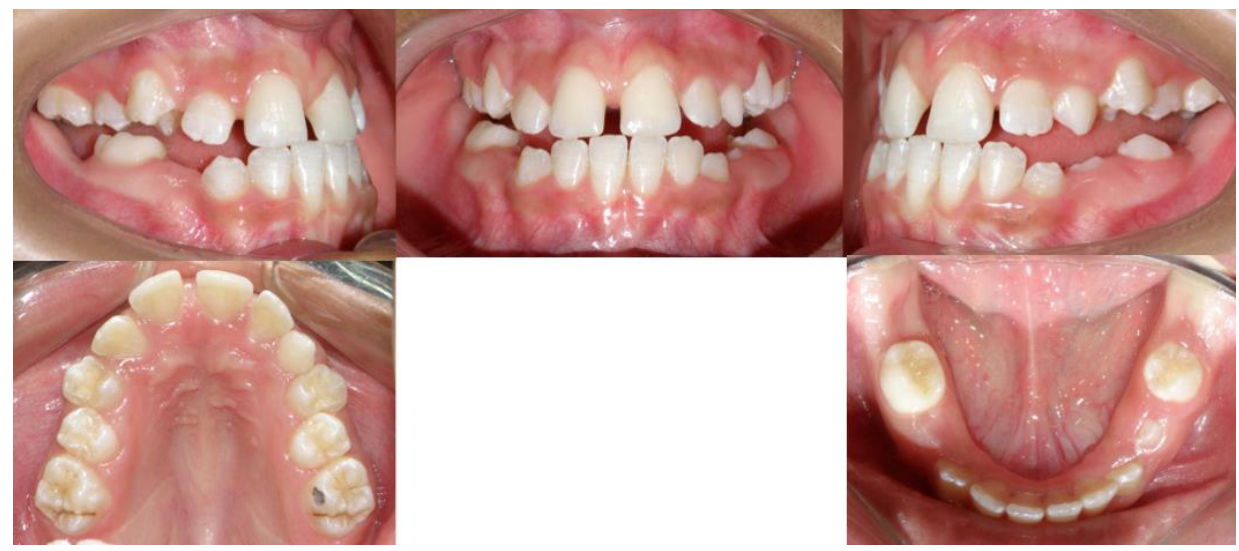

b.
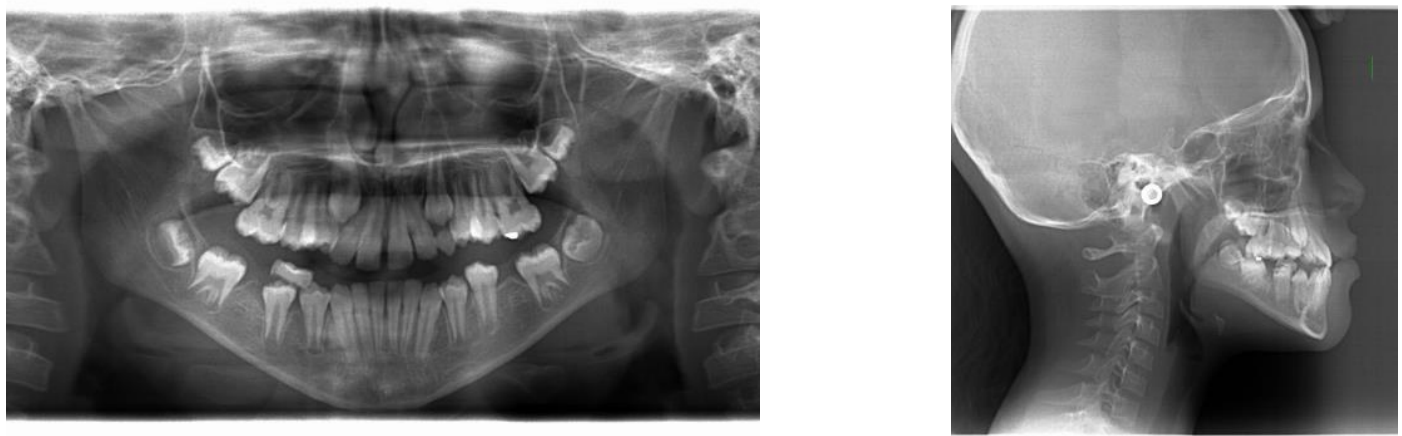

Figura 2. Estudios intermedios.

a. Fotografías intraorales donde se observa la erupción espontánea de los caninos inferiores; b. Radiografía panorámica y de perfil al mes de realizadas las extracciones de los primeros molares mandibulares.

Luego de cinco meses se realizaron tres controles donde se monitoreó el peso y se cuestionó por problemas de deglución o nutrición. La paciente no refirió ningún tipo de inconveniente y tampoco se halló ninguna clase de enfermedad relacionada. Los cambios faciales y esqueléticos fueron mínimos durante estos meses, mientras que los cambios en la posición y vía de erupción de segundos y terceros molares inferiores fueron notables al poco tiempo de evolución. Radiográficamente se observó migración mesial del segundo y tercer molares inferiores bilaterales, así como la erupción espontánea de primeros premolares y caninos inferiores (figura 3). Luego de cinco años, la erupción y alineación de los terceros molares se dio de manera natural; sin embargo, se mantuvo la clase III molar y canina, aunque se logró el objetivo de mantener la inclinación de los incisivos inferiores sin alterar la sobremordida horizontal (figura 4). Un tratamiento posterior de ortodoncia será necesario para la corrección del plano oclusal y mejorar la relación de oclusión posterior. 

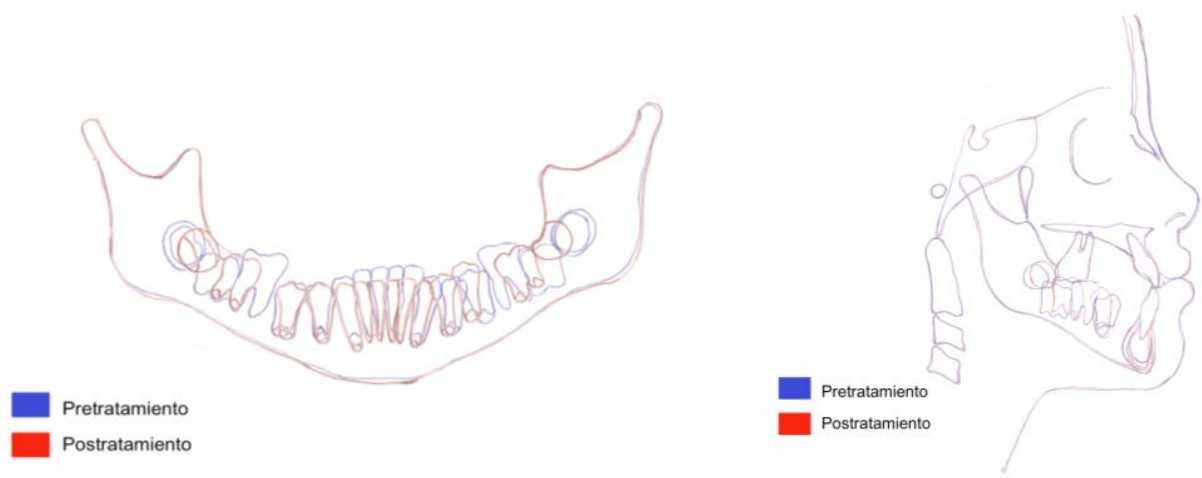

Figura 3. Superposición de radiografías panorámicas y de perfil.

Se observa la migración mesial de segundos y terceros molares mandibulares posexodoncia y el leve cambio esquelético.

a.
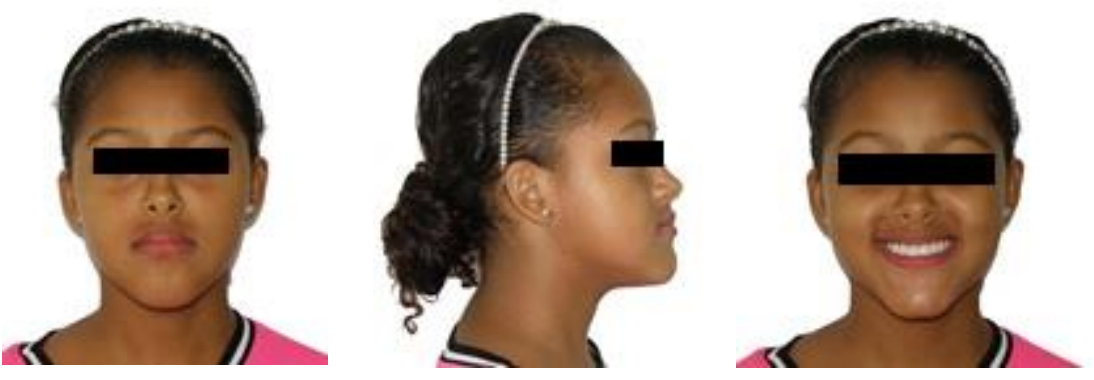

b.

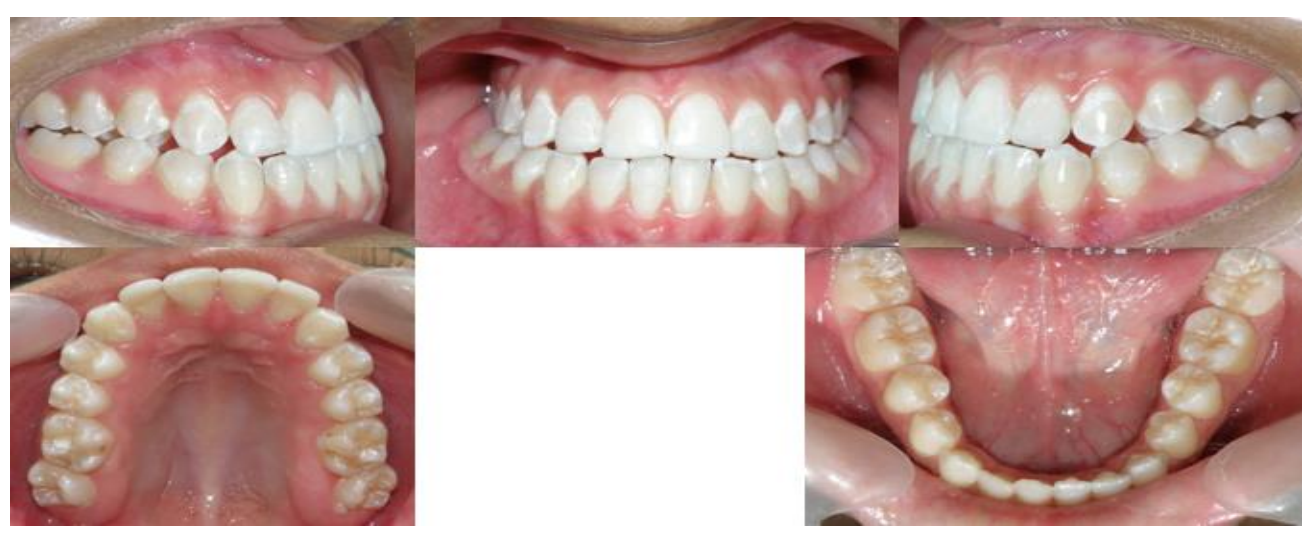

c.
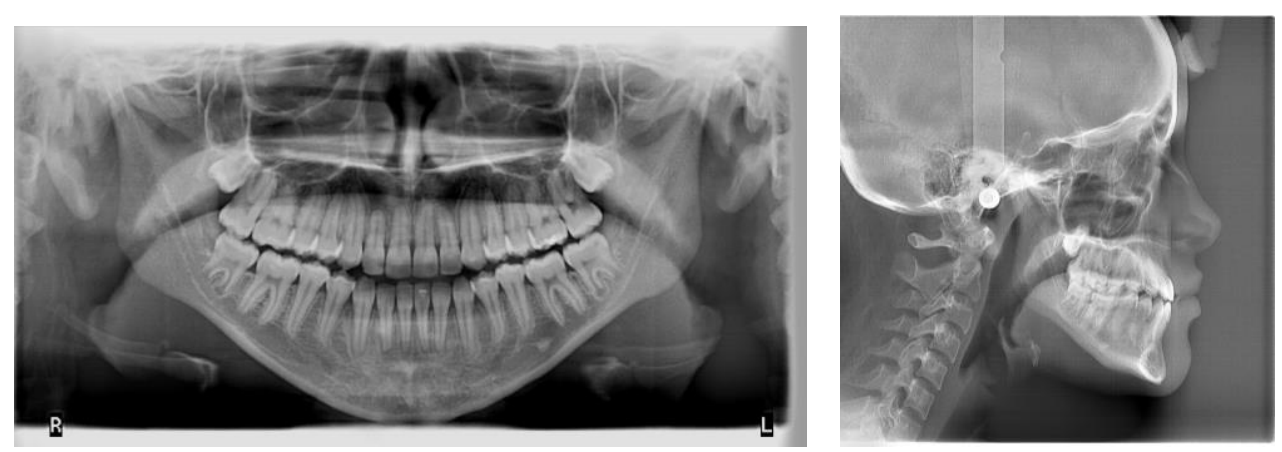

Figura 4. Estudios finales.

a. Fotografías faciales de frente, perfil y sonrisa postratamiento a la edad de 15 años; b. Fotografías intraorales donde se observa la erupción de los terceros molares inferiores; c. Radiografía panorámica y de perfil a los dos años de realizadas las extracciones de los primeros molares mandibulares. 


\section{DISCUSIÓN}

Al igual que en el presente caso, Kondo et $a l^{13}$ deciden, en un adolescente de 12 años de edad con relación esquelética de clase III pero con desviación mandibular hacia la izquierda, y ausencia de primer molar inferior izquierdo, realizar exodoncia del primer molar inferior derecho y, con mecánica ortodóntica, corregir la mordida cruzada anterior y el cierre de espacio posterior utilizando los terceros molares para una adecuada oclusión balanceada. De este modo quedaron los segundos molares inferiores en oclusión con los primeros molares superiores.

Según Stellzig-Eisenhower et $a{ }^{14}$, el análisis de Wits es el principal determinante para definir si un paciente con maloclusión clase III debe ser tratado con camuflaje o con cirugía. Los autores encontraron que para que un tratamiento con camuflaje sea exitoso el análisis de Wits no debe sobrepasar de $-4,6 \pm 1,7$. De igual manera, Burn et $a l^{7}$ en el 2010 reportan camuflajes exitosos de las clases III en pacientes con Wits de -5.0, medida que mejoraba con el tratamiento ya que existió una disminución en la inclinación del plano oclusal y del ángulo SNB, así como extrusión de los molares y un incremento en el plano mandibular. Los anteriores resultados son acordes con el presente caso, que presentó un Wits de $-5,0$, por lo que se espera que los objetivos propuestos se logren con el crecimiento de la paciente y la mecánica propuesta.

Una forma para camuflar las clases III esqueléticas es descrita por Mora et $a l^{15}$, quienes realizan extracciones de segundos molares inferiores en combinación con elásticos intermaxilares de clase III. Los autores encontraron que con este tipo de mecánica se puede obtener una buena relación oclusal sin afectar la estética facial o producir compensaciones dentales marcadas. Además, concluyen que los terceros molares toman la posición de los segundos molares sin mayores complicaciones, por lo que se esperó en el presente caso que los segundos y terceros molares ocuparan el espacio del primer molar sin dificultad.

Como alternativa a los tratamientos de maloclusiones clase III con extracciones se describen casos como el presentado por Ning y Duan $^{16}$ en 2010, donde la terapia incluye extracciones de primeros premolares, o el de
Costa et $a l^{17}$, con extracciones de segundos premolares y el posterior cierre de espacios de extracción. En ambos casos existe una leve retroinclinación de los incisivos inferiores.

Dentro de los tratamientos para las clases III esqueléticas, existen alternativas conservadoras como las descritas por Figueido et $a l^{18}$, que combinan la expansión maxilar con máscara facial y ortodoncia con aparatología fija para lograr una adecuada relación de oclusión sin necesidad de realizar ningún tipo de extracción.

En el 2017 Alkhadra $^{19}$ realiza una revisión sistemática sobre el comportamiento de la oclusión y las implicaciones en los planes de tratamiento cuando se llevan a cabo extracciones de primeros molares durante la dentición mixta. Este autor encuentra así que solo en el $58 \%$ de los casos se da el cierre completo del espacio de extracción $(89,9 \%$ en el maxilar superior y $49 \%$ en el maxilar inferior). Una de las recomendaciones dadas por el investigador para practicar este tipo de extracciones es hacerlas entre los 8-10 años, lo que coincide con la edad en la cual se le realizaron las extracciones de los primeros molares a la paciente del presente caso.

\section{CONCLUSIÓN}

Se presentó el caso de una niña con maloclusión clase III esquelética y apiñamiento severo en el maxilar superior e inferior tratada con extracción de primeros molares mandibulares. Aunque es prematuro conocer los resultados finales y a largo plazo del presente caso, la experiencia sugiere que un correcto diagnóstico que conlleve objetivos específicos de tratamiento puede ayudar a corregir este tipo de anomalía en pacientes en crecimiento activo. La extracción de primeros molares para la corrección de las clases III no es la panacea; es una opción que debe ser evaluada como una forma de tratamiento.

\section{DECLARACIÓN SOBRE CONFLICTOS DE INTERESES}

Somos independientes con respecto a las instituciones financiadoras y de apoyo, y durante la ejecución del trabajo o la redacción del manuscrito no han incidido intereses o valores distintos a los que usualmente tiene la investigación. 


\section{CONTRIBUCIÓN DE LOS AUTORES}

Primer autor: diseño metodológico y análisis estadístico.

Segundo autor: diseño metodológico y análisis estadístico.

Tercer autor: trabajo de campo y redacción.

Cuarto autor: diseño metodológico y análisis estadístico.

\section{REFERENCIAS BIBLIOGRÁFICAS}

1. Plazas J, Martínez O, Castro L, Solana A, Villalba L. Prevalencia de maloclusiones en niños de una escuela en Cartagena de Indias. Ciencia y Salud Virtual. 2011; 3(1): 2-8. Doi: https://doi.org/10.22519/21455333.38.

2. Thilander B, Pena L, Infante $C$, Parada S, de Mayorga C. Prevalence of malocclusion and orthodontic treatment need in children and adolescents in Bogotá, Colombia. An epidemiological study related to different stages of dental development. Eur J Orthod [revista en la internet]. 2001; 23(2): 153-168. Disponible en: https://www.ncbi.nlm.nih.gov/pubmed/1139855 3.

3. Turley P. Treatment of the Class III Malocclusion with Maxillary Expansion and Protraction. Semin Orthod [revista en la internet]. 2007; 13: 143-157. Disponible en: http://www.sciencedirect.com/science/article/pii /S1073874607000266.

4. Rey D, Oberti G, Ealo M, Baccetti T. Camouflage of moderate Class III malocclusions with extraction of lower second molars and mandibular cervical headgear. Prog Orthod [revista en la internet]. 2007; 8(2): 300-307. Disponible en: https://www.ncbi.nlm.nih.gov/pubmed/1803037 6.

5. Lee W, Wong R, Ikegami T. Maxillary second molars extraction. World J Orthod [revista en la internet]. 2008; 9: 52-61. Disponible en: https://www.ncbi.nlm.nih.gov/pubmed/1842610 6.

6. Seon H. Limitations in Orthopedic and Camouflage Treatment for Class III Malocclusion. Semin Orthod [revista en la internet]. 2007; 13(3): 158-174. Disponible http://www.sciencedirect.com/science/article/pii /S1073874607000278.

7. Burns NR, Musich DR, Martin C. Class III camouflage treatment: What are the limits? Am J Orthod Dentofacial Orthop [revista en la internet]. 2010; 137: 9.e1-9.e13. Disponible en: https://dentistry.hsc.wvu.edu/media/1149/classiii-camouflage-treatment-what-are-the-limits.pdf.

8. Baccetti T, Franchi L, Cameron C, McNamara JA. Treatment timing for rapid maxillary expansion. Angle Orthod [revista en la internet]. 2001; 71: 343-350. Disponible en: https://www.researchgate.net/publication/1174 4423_Treatment_Timing_for_Rapid_Maxillary_Ex pansion.

9. Lin J. Lower second molar extraction in correction of severe skeletal class III malocclusion. Angle Orthod [revista en la internet]. 2006; 76: 217-225. Disponible en: https://pdfs.semanticscholar.org/0a7e/148b10c3 c75f09f52bf9a174399f354c2ee0.pdf.

10. Dnyandeo P, Vakil K, Vakil J, Madhukarrao S. Orthodontic Camouflage in skeletal class III malocclusion: a contemporary review. J Orofacial Res [revista en la internet]. 2014; 4(2): 98-102. Disponible

en: http://www.jaypeejournals.com/eJournals/Show Text.aspx?ID=6595\&Type=FREE\&TYP=TOP\&IN= / eJournals/images/JPLOGO.gif\&IID=497\& isPDF=YE S.

11. Carvalho J, Goncalves S, Andrade L, Batista D, Souza R, Almeida V. Accuracy of two cephalometric analysis in the treatment of patients with skeletal class III malocclusion. Brazilian Dent J [revista en la internet]. 2015; 26(2): 186-192. Disponible en: http://www.scielo.br/scielo.php?script=sci_arttex t\&pid=S0103-64402015000200186.

12. Baccetti $T$, Franchi L, Cameron C, McNamara J. Treatment timing for maxillary Expansion. Angle Orthod [revista en la internet]. 2011; 71: 343-350. Disponible en: https://www.ncbi.nlm.nih.gov/pubmed/1160586 7.

13. Kondo $\mathrm{E}$, Ono $\mathrm{M}$, Ahoba TJ. Utilization of third molars in the orthodontic treatment of skeletal class III subject with severe lateral deviation: Case Report. World J Orthod [revista en la internet]. 2004; 5: 201-212. Disponible en: https://www.ncbi.nlm.nih.gov/pubmed/1561233 9. 
14. Stellzig-Eisenhauer A, Lux CJ, Schuster G. Treatment decision in adult patients with Class III malocclusion: orthodontic therapy or orthognathic surgery? Am J Orthod Dentofacial Orthop [revista en la internet]. 2002; 122: 27-38. Disponible en: https://www.ncbi.nlm.nih.gov/pubmed/1214289 4.

15. Mora M, Pesqueiro R, Hernández G, de Silva J, Rodríguez J. Alteración Dentofacial clase III tratado con camuflaje. Rev Mex Ortod [revista en la internet]. 2015; 3(1): 62-69. Disponible en: https://www.sciencedirect.com/science/article/p ii/S2395921516000180.

16. Ning F, Duan Y. Camouflage treatment in adult skeletal class III cases by extraction of two lower premolars. Korean J Orthod [revista en la internet]. 2010; 40(5): 349-357. Disponible en: https://ekjo.org/search.php?where=aview\&id=10.4041/kj od $.2010 .40 .5 .349 \&$ code $=0123 \mathrm{KJOD} \&$ vmode $=$ PUB READER.
17. Costa TM, Ustrell JM, Correia, J. Orthodontic camouflage in the case of a skeletal class III malocclusion. World J Orthod [revista en la internet]. 2004; 5: 213-23. Disponible en: https://www.ncbi.nlm.nih.gov/pmc/articles/PMC 5029321/.

18. Figueido $M$, Furquim $D$, Bommarito $S$, Scanavini M. Orthodontic compensation in skeletal class III malocclusion: a case report. World J Orthod [revista en la internet]. 2007; 8: 385-396. Disponible en: https://www.researchgate.net/publication/5755 596_Orthodontic_compensation_in_skeletal_Clas s_III_malocclusion_A_case_report.

19. Alkahadra T. A Systematic review of the consequences of early extraction of first permanent first molar in different mixed dentition stages. J Int Soc Prevent Communit Dent [revista en la internet]. 2017; 7(5): 223-226. Disponible en: https://www.ncbi.nlm.nih.gov/pubmed/2902669 2. 